\title{
A simple blue light pulse generator with GaN/SiC Light Emitting Diodes for the time response testing of PMTs
}

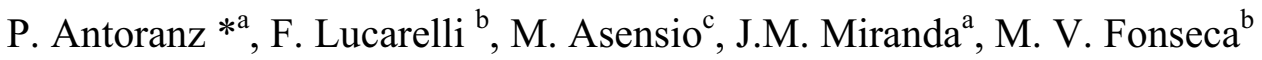 \\ ${ }^{a}$ Dpto. Física Aplicada III, Universidad Complutense de Madrid \\ ${ }^{\mathrm{b}}$ Dpto. Física Atómica, Molecular y Nuclear, Universidad Complutense de Madrid \\ ${ }^{c}$ Dpto. Infra., I. Sistemas Aeroespaciales y Aerop., Universidad Politécnica de Madrid
}

\begin{abstract}
A simple and cost-effective integrated synthesizer of fast light pulses has been designed, analyzed and tested for the characterization of the time response of photo-multipliers (PMT). This synthesizer consists of an integrated pulse generator based on Schmidt Trigger Inverters, a broadband matching network and a high speed LED. It enables the generation of pulses as short as less than $10 \mathrm{~ns}$ with variable pulse width, amplitude and repetition frequency. In order to accurately know the shape of the pulses applied to the PMT under test, a circuital model of the LED has been developed and verified at frequencies up to $2 \mathrm{GHz}$. This model accounts for the nonlinear behavior of the LED capacitance as well as the package parasitics. The influence of the mismatch at the different frequency components of the synthesized pulse has been investigated. The pulse transmitter has been used to test the time response of MAGIC telescope pixels.
\end{abstract}

Keywords: Gamma-ray astronomy detectors, hysteresis, pulse generation, impedance matching, light-emitting diodes, photomultipliers, schmitt trigger

\section{INTRODUCTION}

The Major Atmospheric Gamma-ray Imaging Cherenkov (MAGIC) Telescope is devoted to international colaboration devoted to the observation of the Cherenkov Radiation present in the atmosphere ${ }^{1}$. When cosmic rays penetrate into the air, they interact with the nuclei of the atoms thus emitting a shower of charged secondary particles. The velocity of those particles can be higher than light speed, therefore producing the cone-shaped light emission called Cherenkov Radiation. ${ }^{2}$ The intensity of this radiation is proportional to its frequency, so the shorter the wavelength of the emission, the bigger the amplitude of the wave. The detection in the visible range of energies is consequently optimum in the blue range of the spectrum. By analysing this blue light it is possible to better understand the high energy particles that cause the initial showers, otherwise very difficult to detect because of their high energy and their lack of charge (and not being, therefore, affected by magnetic fields). Some sources of this high energy particles can be pulsars, Active Galaxy Nuclei (AGNs), Gamma Ray Bursts (GRBs), etc. Cherenkov pulses arrive randomly following a Poisson distribution with a mean frequency around $200 \mathrm{~Hz}$. Time differences between pulses can thus be arbitrarily short. The typical pulse width of Cherenkov light produced in atmospheric showers is of a few ns. ${ }^{3}$

The detection system of the telescope consist on an array of mirrors, conforming a paraboloid with a diameter of 17 $\mathrm{m}$ and $239 \mathrm{~m}^{2}$ of area. The incident light is reflected towards the camera placed in the focus, which consists on a matrix of 577 pixels (detectors). ${ }^{4}$ In the current telescope, the sensors used are photomultiplier tubes (PMTs). ${ }^{5,6}$ The construction of a second telescope, with a camera composition still under development, has already been planned. ${ }^{7,8}$ Some devices are being tested, as new generation of PMTs or hybrid photodetectors (HPDs). ${ }^{9}$ Regardless of the type of

*antoranz@gae.ucm.es; phone+3491394 4491 
sensor used, the pulse generator implemented for the tests must satisfy the following requirements: (1) The system must be fast enough to achieve the study of the shortest pulses of Cherenkov radiation. (2) The light intensity must be variable, as the pixels could need different signal amplitudes for a correct calibration. The pulse must be driven into a high luminosity blue LED, so deep studies and models of such light sources are not irrelevant. (3) The discontinuities in the transition between the generator and the LED must be minimized, in order to avoid distortion, delays or other width increments into the pulse shape. A study of the different types of connectors must be done, and the possibility of coupling nets or output buffers should be contemplated. (4) Noise must be studied in order to distinguish the one produced by the sky background when real measurements are done from that produced by the amplifier. It should be possible to reciprocally insulate the electronic of the telescope and the pulse generator. (5) It must be as reliable as possible, as the maintenance is more complicated in the telescope than in an ordinary lab. (6) The integration into a software controlled chain should be feasible, therefore simplifying the routine pixel calibration. (7) Weight, size and power consumption must be as reduced as possible, in order to minimize space and the mechanical forces needed when included in the onboard telescope instrumentation.

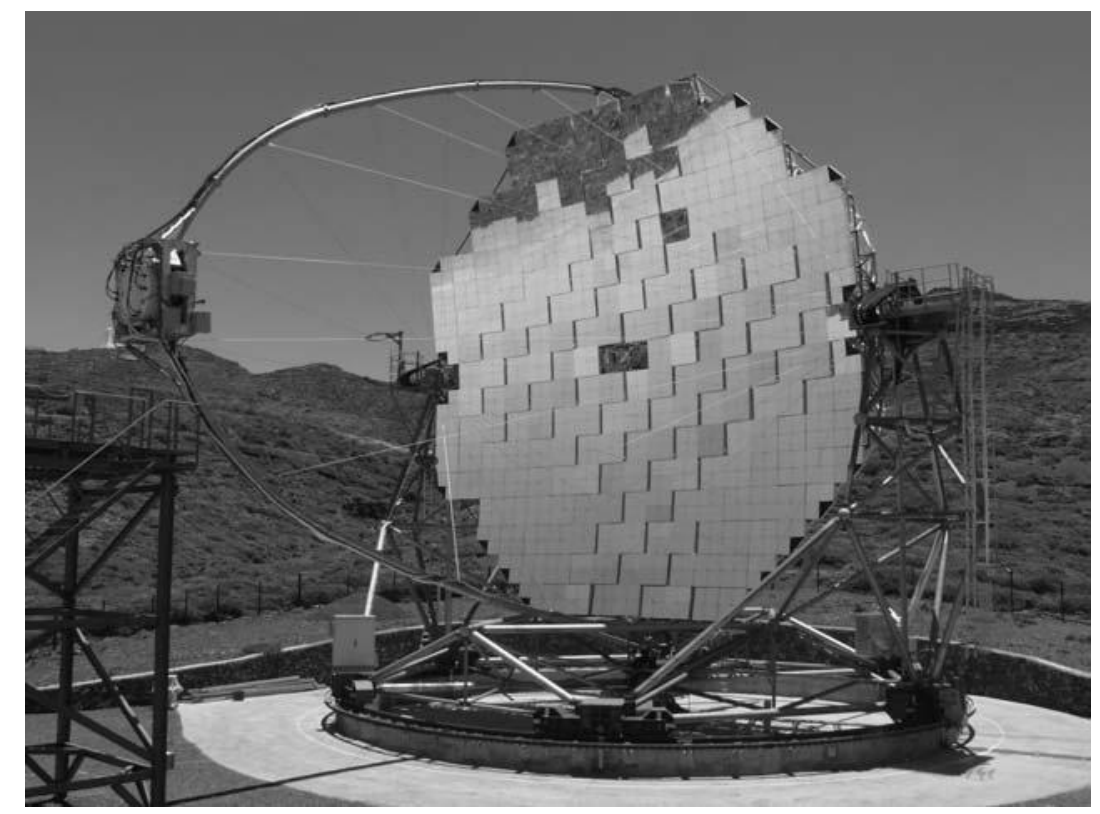

Figure 1. General onsite view of the MAGIC Telescope.

\section{PULSE GENERATION}

\subsection{The Schmitt Trigger.}

The theoretical basis of the developed pulse generator lays on the well known hysteresis behaviour of the Schmitt Trigger inverter. ${ }^{10}$ As shown in Fig. 2, the asymmetry in the characteristics provide the generation of a square wave when the charge and discharge of a capacitor placed at the input is forced by means of a feedback resistance. The capacitor is initially discharged, so the input of the inverter is 0 and the output is 1 . The current flows then through the resistance and begins to charge the capacitance, until the value $V_{I H}$ is reached. At that moment, the output turns to 0 , and the current changes its direction discharging the capacitor until $V_{I L}$, when the output changes to 1 . The process is repeated once and again while the inverter is polarized, thus generating this way a symmetric square wave. The amplitude can easily be controlled by means of gate polarization $\left(V_{O H} \approx V_{C C}\right)$ or by a potentiometer placed at the output, and the frequency by means of the time constant RC. Figure 3 shows the capacitor curve (a), and the square wave generated (b). 
The main difficulty of this step is to achieve a good time response, that is, to minimize the rise and fall time of the wave edges. Among the variety of Schmitt Triggers commercially available, the faster one belongs to the CMOS family (74AC14). This technology is the one that best suits for the present purposes, as the power consumption is minimized (the quiescent supply current of the device is very small, up to a few $\mu \mathrm{A}$ ) and the edges are symmetrical and as a pair of ns short. ${ }^{11}$
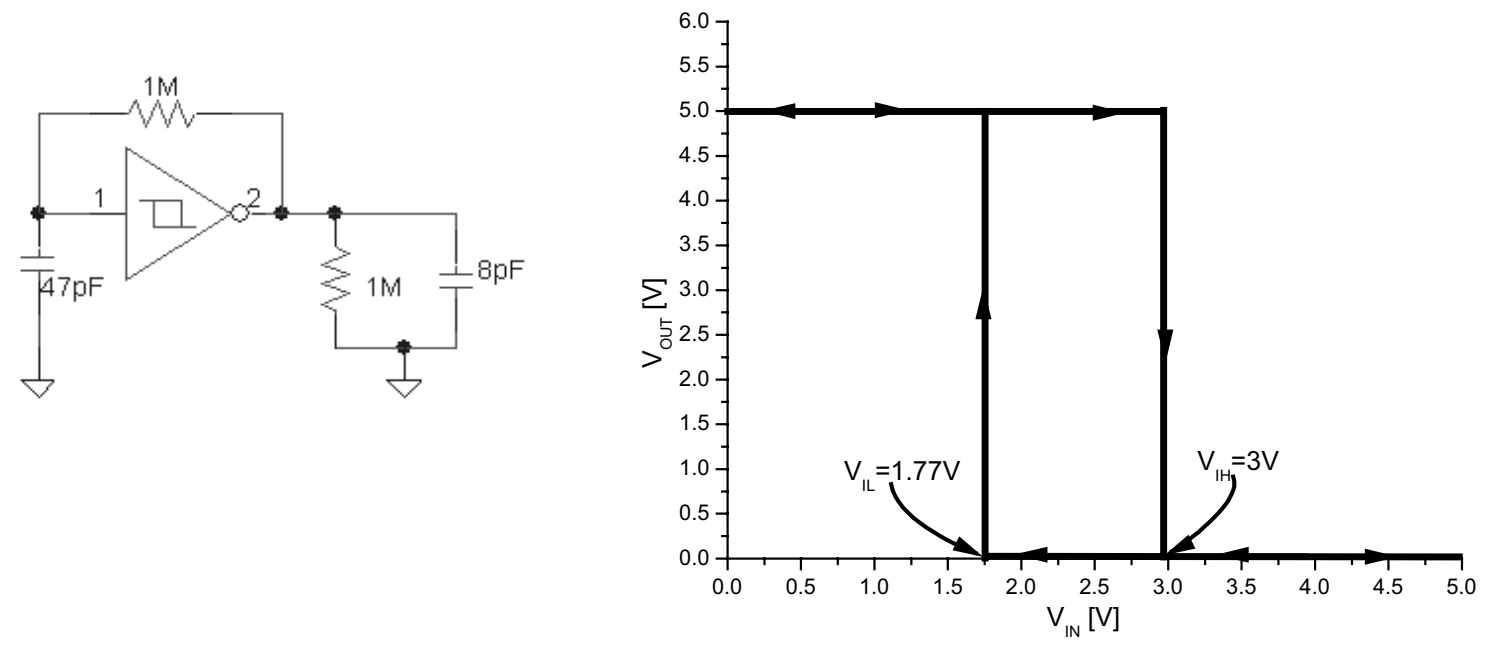

Figure 2. (a) Square wave generator. (b) Hysteresis curve of the 74AC14 HEX inverter.
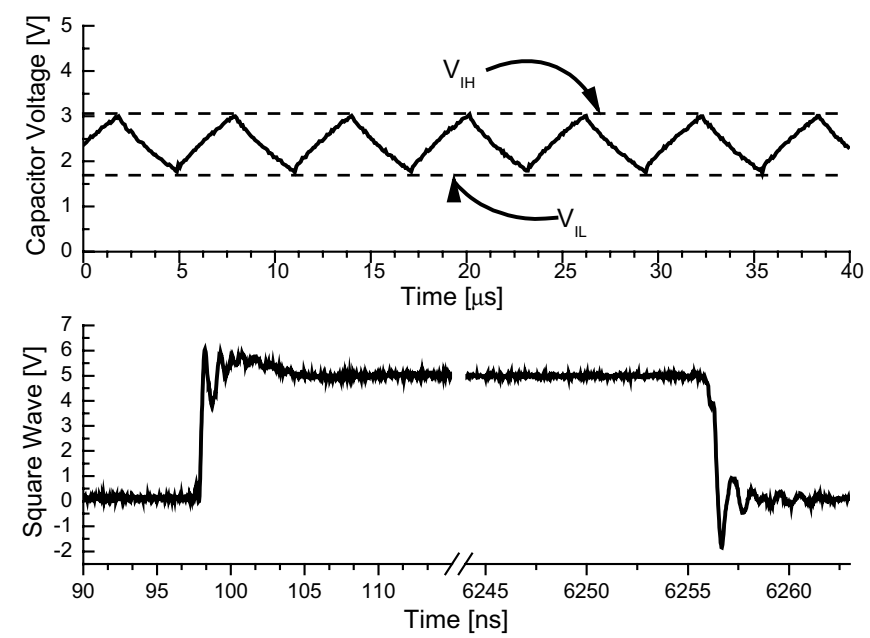

Figure 3: (a) Charge and discharge of the capacitor at the input of the inverter. (b) Rise and fall edges of the square wave.

\subsection{Pulse shaping.}

The straightforward step is to reduce the square wave into a very narrow pulse. Two methods have been studied for that purpose: 


\subsubsection{Different paths for charge and discharge of the capacitor.}

The path established for the charge of the capacitor sets the frequency of the pulsed wave as explained above, while the discharge path is set with a new resistance, as low as possible. Thus, the low to high transition of the input (high to low at the output) determines the frequency, and the high to low, which ought to be as fast as possible, determines the duty cycle and thus the pulse width. It is to be remarked that the obtained pulse is negative. The first stage of Fig. 4 (a) shows this implementation, and Eq. (1), (2) the time constants:

$$
\begin{aligned}
& \tau_{\text {charge }}=R_{\text {charge }} C \\
& \tau_{\text {discharge }}=R_{\text {discharg } e} C
\end{aligned}
$$

where:

$$
\begin{aligned}
& R_{\text {charge }}=1 M \Omega \\
& R_{\text {disch arge }}=\left(15 \Omega+R_{D}\right) / / 1 M \Omega \approx\left(15 \Omega+R_{D}\right) \\
& R_{D}=r_{D}+R_{S}
\end{aligned}
$$

Included in the discharge time constant is the resistance of the diode under direct bias $R_{D}$ : the dynamic resistance $r_{D}$, and the parasitic series resistance of the depletion layer and wire bonding $R_{S}$. While the former effect is negligible, the latter can add some tens of ohms to the discharge resistance, ${ }^{12}$ therefore affecting the pulse shape.
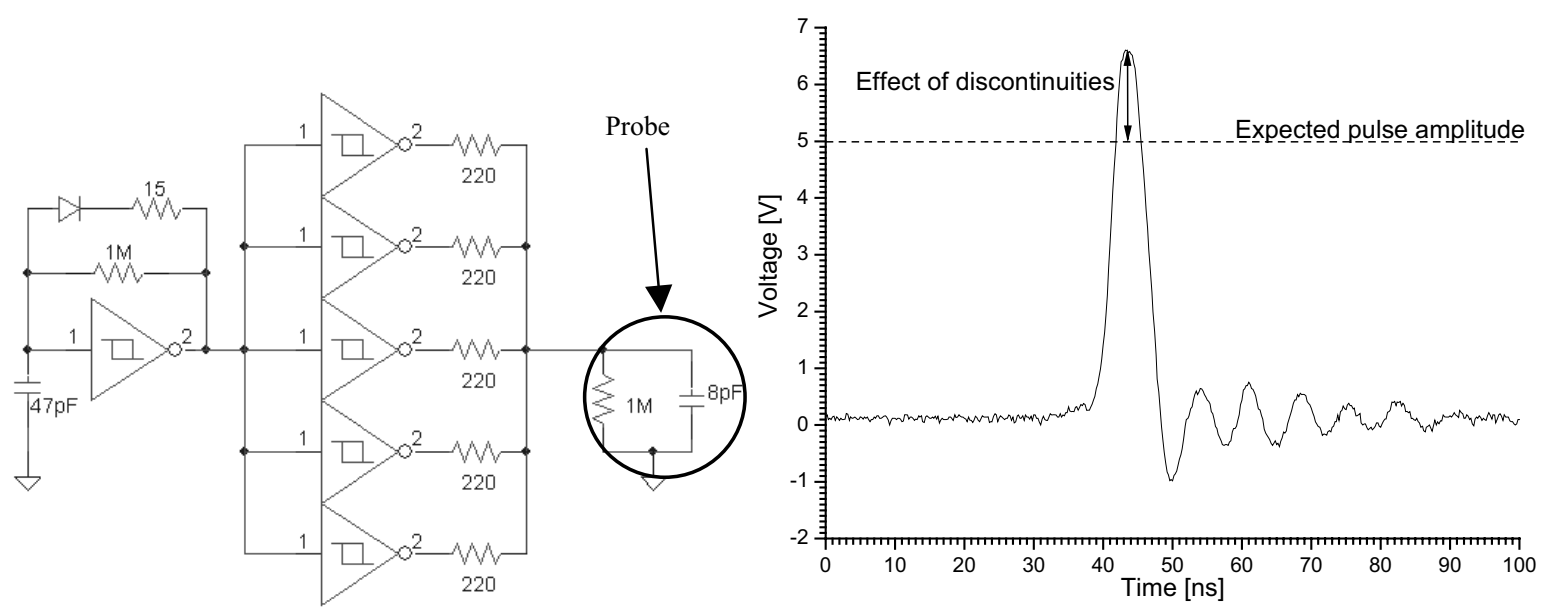

Figure 4. (a)Pulse generator with output buffer. (b) Generated pulse, measured with the oscilloscope in open circuit (the probe has a resistance of $1 \mathrm{M} \Omega$ and a capacitance of $8 \mathrm{pF}$ ) Some ringing is produced.

In order to isolate the capacitor from the LED, and to invert the pulse to obtain a positive one, an output inverter buffer is used. It consists in five more inverters (the 74AC14 provides six gates per chip), which internal impedance, combined with $220 \Omega$ series resistance provides an output path of $50 \Omega$ (see Fig. 4 (a)). The convenience of implementing a matching network to couple the output impedance of the system to the input impedance of the blue LED will be discussed later. The resulting pulse is shown in Fig. 4 (b). It has been sampled with an oscilloscope probe with a $1 \mathrm{M} \Omega$ resistance and a $8 \mathrm{pF}$ capacitance, so it could be considered as an open circuit and the pulse as the one that will excite the LED. It can be seen the effect of discontinuities: some ringing is produced, what causes the pulse to be higher than 
expected. The effect of the LED when connected at the output, and its relation with the observed distortion of the signal will be studied in following sections.

\subsubsection{Delays of the logic gates.}

If the 'AND' function of a square wave an its inverted one is done, the result would be a logical zero. But as a certain delay exists in the propagation of the signal through each gate components, a 'glitch' will occur at the output of the AND gate (Fig. 5 (a)). This results in an output pulse, with an ideal amplitude of $V_{C C}$ (logic gate bias), and a width that equals the delay of the inverter gate. Values of 5-7 ns have been measured in this delay. As shown in Fig. 5 (b), the behaviour is not ideal since rise and fall times appear a well as some ringing are present. These effects will affect the generated pulse as shown (reduced amplitude and higher pulse width). The signals have been measured with the oscilloscope probe described above.
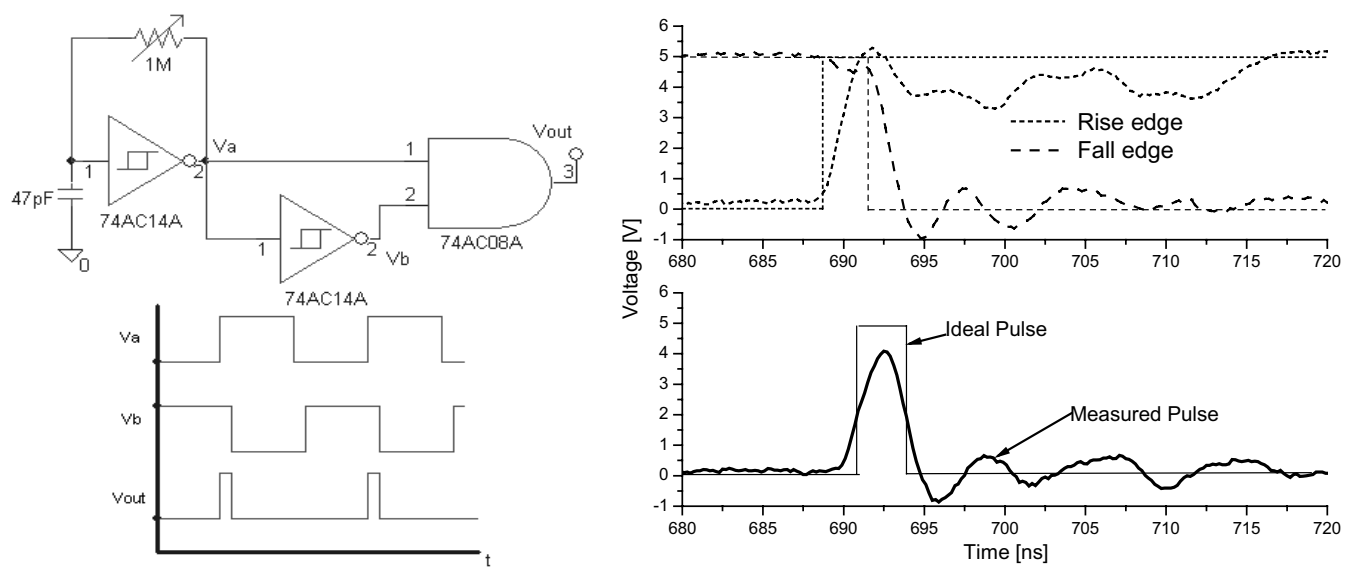

Figure 5. (a) Pulse generator based on the logical glitches (ideal response). (b) Actual generated pulse.

The pulses obtained with this generator are even shorter than in the previous case. However, the power consumption and the number of active devices (and thus the possibilities of device failure) increases, so the previous design has been selected to do the sensor testing.

\section{LED MODELING}

A well behaved electrical pulse signal displayed in an oscilloscope does not necessary ensure that the RF pulse generator will provide a well behaved optical pulse when connected to the LED. Mismatches between the generator and the device typically result into signal losses and pulse ringing. An accurate bias dependent model of the LED is therefore necessary to account for these effects.

A number of factors must be accounted for when simulating the LED under pulsed operation. Modelling the impedance of a blue LED is not as simple as modelling a standard one. Even the ideality factor of a blue LED in a DC curve does not behave as one should expect from the standard theories. ${ }^{13,14}$ On the other hand, modelling the AC behaviour poses additional difficulties. When direct polarization is applied, neither the input capacitance nor the dynamic resistance are constant but both depend on bias 
To understand these effects and to adequate the optical signal to our needs, a semi-empirical model of blue LEDs. The equivalent circuit is shown in Fig. $6 .{ }^{15}$ It includes two parasitic elements $C_{p}$, and $L_{p}$. The former simulates the capacitance of the device package, the latter the inductance of the bonding wires. The other $\left(R_{S}, C_{j}, R_{j}\right)$ account for the intrinsic behaviour of the LED. The subminiature A (SMA) connector in which the devices were assembled is simulated with a short section of a coaxial transmission line, with a measure length of $7.3 \mathrm{~mm}$. An impedance analyser HP4192A and a network analyser HP8720C have been used to extract such parameters in the range of voltages $(0-5 \mathrm{~V})$ and up to frequencies $(2 \mathrm{GHz})$ which cover the pulse characteristic bandwidth.

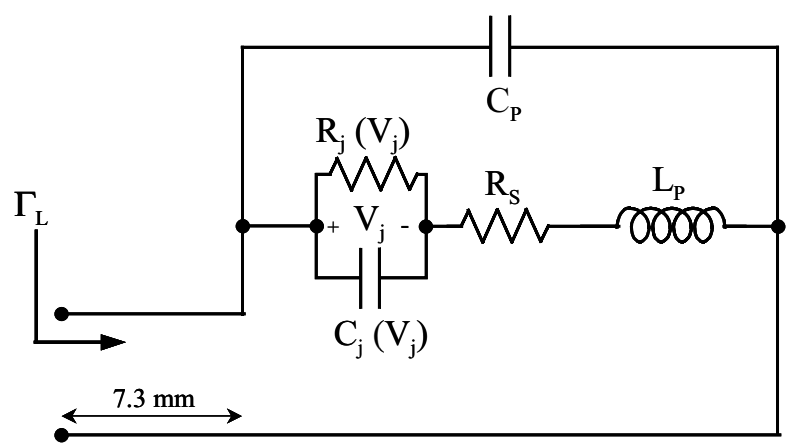

Figure 6. Equivalent circuit of the LED

The proposed model for the blue LEDs was verified with measurements of devices mounted on standard T1 3/4 (5 $\mathrm{mm}$ ). Accurate estimation of the device impedance were obtained even at frequencies at which this impedance is fully dominated by parasitics. The parasitic inductance of the bonding wire was identified as the main limiting factor in the high frequency behaviour of the device. ${ }^{15}$ In addition, the relatively large series resistance of these LEDs, between 13 and $25 \Omega$, makes the diode impedance close to the generator one. Therefore the model predicts small ringing effects.

\section{TRANSITIONS AND MATCHING NETWORKS}

Once the LED has been analysed, the input impedance presented to the applied pulse can be estimated. Classical procedures of impedance matching based on narrow band reactive elements cannot be applied to couple a LED to a pulse generator due to two main reasons: a) the pulse signal has a wide frequency bandwidth, and b) the LED impedance is bias-dependent. However, dissipative coupling networks can reduce reflections and therefore ringing at the expense of a slight signal loss. In this case a trimmer series resistance could be placed at the output of the generator and in series with the diode.

Figure 7 shows the pulse shape measured at the diode pins, for three values of the mentioned resistance. The signal loss can be considered acceptable for coupling resistances below $20 \Omega$. Above this value, the peak voltage applied to the LED is too close to its threshold voltage. However, the observed residual ringing levels are far below the LED turn on voltage, as one could expect from the simulation, and therefore they are not replicated in the optical signal. Consequently, no matching networks were considered necessary for the tested devices. 


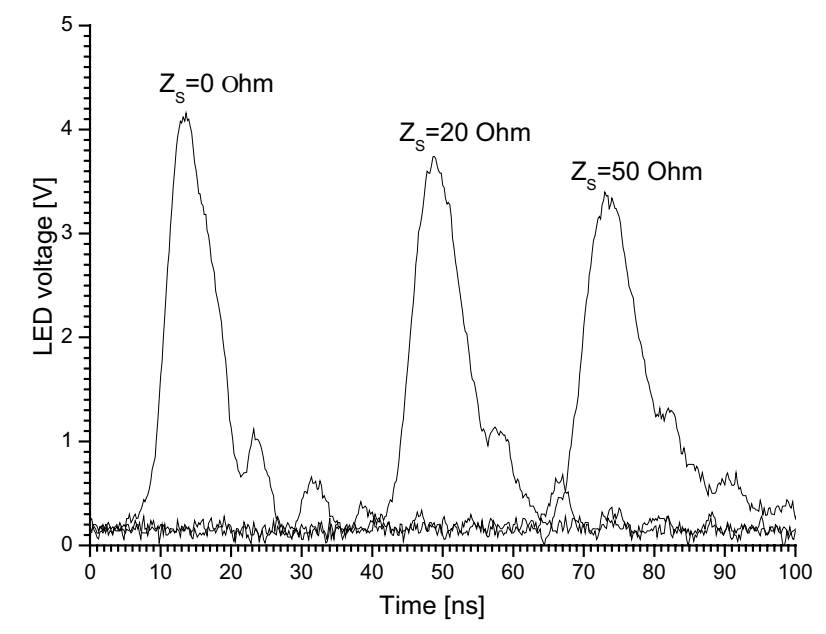

Figure 7. Effect of the addition of a series resistant to the LED connected at the output of the pulse generator.

\section{PULSE DETECTION}

Once the pulse set up is done, and the electrical behaviour of the LED is known, the next step is to test the optical response of the LED and the detector. The generator-pulse chain is polarized and placed inside the black box $(1 \times 0,5 \mathrm{x}$ $0.5 \mathrm{~m}$ ) used to test the sensors. The device used to characterize the optical response of the generator was a photomultiplier (Hamamatsu EMI9116) ${ }^{5,6}$ which is already installed in the current Telescope camera.

It is known that the photomultiplier response is very fast $(1.3 \text { to } 5 \mathrm{~ns} \mathrm{FWHM})^{6}$, so the generated light pulse must induce a clear and well shaped pulse in the detector, whose rise and fall time correspond to the rise and fall time of the light intensity of the LED. The signal amplitude is somehow more difficult to estimate, because of the many parameters involved (quantum efficiency (QE) of the photomultiplier, angular distribution of the LED emission, ${ }^{16}$ distance and solid angle comprised by the detector window with respect to the light source, etc) and is not relevant for the interests of this work.

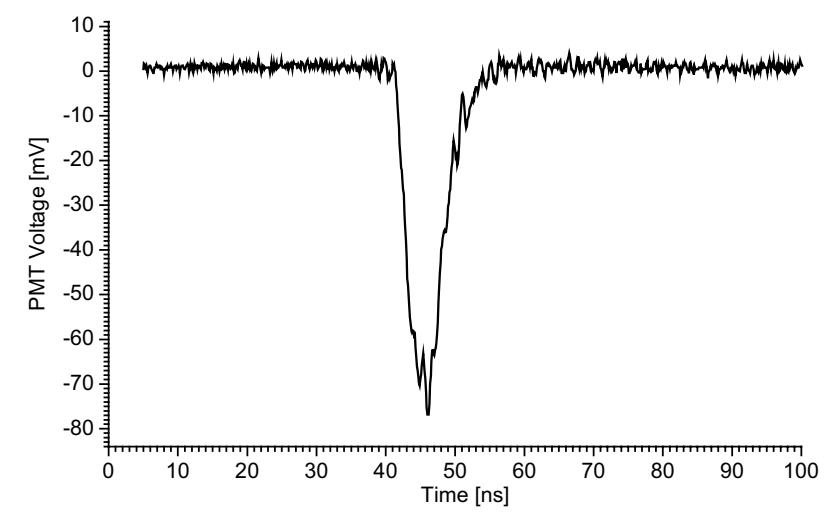

Figure 8 . Pulsed light detected by the photomultiplier tube

It should be stressed that the noise produced by the pulse generator is not relevant. None of the blue LEDs is turned on until the polarization reaches about $2.5 \mathrm{~V}$, so the noise coming from the devices, and even the ringing produced by the discontinuities are never by far optically transmitted to the detectors. Therefore, the only noise to care about in real 
working conditions are the one due to the intrinsic characteristics of the detector, and the one produced by sky background, both treated in the data acquisition.

A new testbench for HPD is being developed by the authors, which will allow further optical detection tests. The generator-HPD chain will be used to test the response of several high speed amplifiers for future hardware configurations of the Telescope camera.

\section{CONCLUSIONS}

In order to test the ultra fast light detectors used in the MAGIC telescope, a high speed pulse generator has been developed. Two different models have been designed, by means of the hysteresis characteristics of the Schmitt Trigger inverters. Both of them have been tested and proved to reach pulse widths up to 4 ns, which is by far enough to reproduce the work conditions of the detectors.

The pulse amplitude must be controlled because of the high sensitivity of the photodetectors. HPDs are especially sensitive, so high intensity light could produce irreversible damages. This implies that the tests must be done in dark conditions, and that the light of the LED must be controlled. The complete chain is enclosed into a small black, and the polarization range of the blue LEDs is set between 2.5 and $5 \mathrm{~V}$, what can be achieved by varying the dc bias of the inverters. This produces a small widening of the pulse, but never exceeding 6-7 ns wide. By means of a software controlled voltage source, the light emission intensity can be remotely controlled.

The effect of discontinuities has been studied. To connect the generator to the LED a SMA connector ( $50 \Omega$, up to 6 $\mathrm{GHz}$ ) has been used. A semi-empirical model of the LEDs used has been developed to estimate the input impedances. The expected values are close to $50 \Omega$, what is experimentally confirmed when coupling the LED to the generator by means of a matching network.

Noise effects have been discarded. Out of the pulse region, the noise is typically the corresponding to the measuring devices, due to the fact that the signal is ideally always 0 (only observing the ringing due to mismatching and parasitics). Anyway, the noise outside the peak maximum is not of much importance, because even in the worst conditions the noise amplitude is never enough to turn the LED on, and thus to be transmitted to the photomultiplier or HPD. This implies that all the noise measured in real working conditions will be due to the sky background and not to the electronics.

Surface Mounting Devices (SMD) have been used in the final implementation, so the size and weight of the generator are extremely reduced. Thist makes it suitable for the use inside the black box or other reduced testbench. Only two types of active devices (the inverters and a diode) are necessary and the working conditions (temperature, bias...) are always below the maximum recommended. Therefore, the lifetime expected is the one provided by the device manufacturer, making the complete system very reliable.

The complete chain (pulse generator - LED - photomultiplier) has been tested, and the fast response of the photomultiplier has been proved. With the completion of this cost-effective pulse generator, further analysis are planned, including a new testbench for HPDs and high speed amplifiers design and test, which could be in the future integrated in the signal transmission chain.

\section{AKNOWLEDGES}

This paper has been partially supported by CICYT (project FPA2000-1802-C02-01). We also acknowledge Razmik Mirzoyan, Eckart Lorenz and Manel Martínez for their valuable technical support. 


\section{REFERENCES}

1. M.V.Fonseca, The MAGIC telescope project, Acta Physica Polonica B, vol. 30, pp. 2331 (1999).

2. W.R.Leo, Techniques for Nuclear and Particle Physics Experiments, Springer-Verlag, Second Edition, pp 35-37.

3. Hoffman et al., Gamma-ray astronomy at high energies, Rev. Mod. Physics., vol 71, No. 4, July 1999, pp 897-936.

4. J.A.Barrio et al, The MAGIC telescope, design study for the construction of a $17 \mathrm{~m}$ Čerenkov telescope for GammaAstronomy above 10Ghz, MAGIC Proposal, December 1997, pp 43-92

5. F.Lucarelli, M.Cámara, P.Antoranz, J.M.Miranda, M.Asensio, J.A.Barrio, M.V.Fonseca, Testbench to characterize the pixels of the MAGIC Telescope IEEE Transactions on Electron Devices, submitted for publication.

6. PMT Handbook (Complete), HAMAMATSU http://sales.hamamatsu.com/assets/applications/ETD/pmt_handbook/pmt_handbook_complete.pdf

7. E.Lorenz, H-J.Gebauer, D.Kranich, M.Merk, R.Mirzoyan, Progress in the development of a high QE, red extended photomultiplier for the second phase of the MAGIC telescope, Nuclear Instruments and Measurements in Physics Research A, vol. 504, (2003), pp. 280-285

8. J.Gebauer, D.Ferenc, D.Kranich, E.Lorenz, M.Merk, R.Mirzoyan, Evaluation of a new high QE photomultiplier for air Cerenkov telescopes, Nuclear Instruments and Measurements in Physics Research A, vol. 518, (2004), pp. 615618

9. C.D'Ambrosio, H.Leutz, Hybrid photon detectors, Nuclear Instruments and Measurements in Physics Research A, vol. 501, (2003), pp. 463-498

10. Thomas A.DeMassa, Zack Ciccone, Digital integrated circuits, John Wiley and Sons, 1996, pp 316-322.

11. 74AC14 Datasheet.

12. P.Antoranz, J.M.Miranda, F.Lucarelli, M.Cámara, M.Asensio and M.V.Fonseca, A DC Model of Blue Light Emitting Diodes Including Thermal Effects, IEEE Electron Device Letters, submitted for publication.

13. Jay M.Shah, Y.-L. Li, Th. Gessmann, and E.F.Schubert, Experimental analysis and theoretical model for anomalously high ideality factors $(\eta>>2.0)$ in AlGaN/GaN p-n junction diodes, Journal of Applied Physics, Vol. 94, No. 4, 15 (2003)

14. J.T.Torvik, M.Leksono, J.I.Pankove, Optical characterization of GaN/SiC n-p heterojunctions and p-SiC, Applied Physics Letters, Vol 72, Issue 8, pp. 945-947 (1998)

15. P.Antoranz, J.M.Miranda, J.L.Sebastián, M.Cámara and V.Fonseca, High frequency modeling of GaN/SiC blue light emitting diodes, Journal of Applied Physics, to be published.

16. M.Cámara, F.Lucarelli, M.V.Fonseca, I.Oya, P.Antoranz, J.M.Miranda, An analytical model of a LED-based transmission system for the test of highly sensitive photodetectors, American Journal of Physics, submitted for publication. 